\title{
Asymptotic Stability and Asymptotic Synchronization of Memristive Regulatory-Type Networks
}

\author{
Jin-E Zhang \\ Hubei Normal University, Hubei 435002, China \\ Correspondence should be addressed to Jin-E Zhang; zhang86021205@163.com \\ Received 31 October 2016; Accepted 10 January 2017; Published 26 January 2017 \\ Academic Editor: Xin Yu \\ Copyright (C) 2017 Jin-E Zhang. This is an open access article distributed under the Creative Commons Attribution License, which \\ permits unrestricted use, distribution, and reproduction in any medium, provided the original work is properly cited. \\ Memristive regulatory-type networks are recently emerging as a potential successor to traditional complementary resistive switch \\ models. Qualitative analysis is useful in designing and synthesizing memristive regulatory-type networks. In this paper, we propose \\ several succinct criteria to ensure global asymptotic stability and global asymptotic synchronization for a general class of memristive \\ regulatory-type networks. The experimental simulations also show the performance of theoretical results.
}

\section{Introduction}

Using memristive devices as synapses is a focus in memristive networks. To extract the benefits of high-efficiency memristive memory, various memristive networks have been reported to date [1-18]. Unlike conventional two-terminal devices, memristive networks exhibit pinched memristor hysteresis loop characteristics, making them particularly suitable for linear-drift devices [10]. Moreover, as the modular compact model for memristors, memristive regulatorytype networks are further broadened to memristive systems that exhibit the phenomenon of closed-form sneak paths, which enable nanoscale geometries with short access latencies. A memristive regulatory-type network contains multiply-threshold synapses, which has been heralded as a new paradigm in large-scale circuits. Compared with some memristive systems, a memristive regulatory-type network has the following advantages: (1) it is more biomimetic in behaviors with simple system structure; (2) it simplifies the structure and complication of circuits and is easy to realize. With these coveted properties, memristive regulatory-type networks have the potential of realizations in module-based nanoscale neuromorphic computing systems.

The underlying physics mechanism of memristor models is extremely complex. In order to explore the characteristics and applications of memristive networks, several attempts in $[1-4,6-18]$ have been made, using nonlinear system theory, to develop behavioral models of memristors. An ideal dynamic property is a critical requirement for the development and validation of memristive networks. Evolutional characteristics of memristive networks are an interesting and prosperous research area. However, deploying nonlinear analysis technology in memristive networks is challenging because a memristive network is basically a switched network cluster $[13,15]$. Such switched network cluster thus possesses the synaptic action, in which the synaptic weight can be incrementally ameliorated by adjusting the charge or flux through it. There are two major obstacles to analyze and control the memristive networks, namely, high complexity and switched hybridity $[12,13]$. On the other hand, dynamical analysis for memristive networks can explain carrier dynamics and associated transients. Once the electronic properties of memristive networks are revealed, then the circuit models can be implemented based upon the underlying dynamic nature. By tweaking physical structures and bias conditions, system designer can optimize the circuit performance, and then, numerous potential applications of the memristors have been exploited, such as neuromorphic, digital, and quantum computation.

In spite of having significant progress in the area of nonlinear control systems [19-35], memristive regulatorytype networks constituting switched network cluster have received less attention. It has been reasoned that much like neuroevolutionary systems, memristive regulatory-type 
networks could be responsible for different neuromorphic architectures [36, 37]. To this end, we focus on the evolution of memristive regulatory-type networks. In this paper, we study global asymptotic stability and global asymptotic synchronization of a class of memristive regulatory-type networks. Based on $M$-matrix theory, we develop less conservative global asymptotic stability results and global asymptotic synchronization results for memristive regulatory-type networks. Such theoretical analysis can significantly help understand and identify system performance, especially in neuromorphic computing era where stability or synchronization is crucial. In fact, dynamic analysis of memristive regulatorytype networks can provide an overview for optimizing the circuit device and enhancing circuit performances.

The rest of this paper is organized as follows. Section 2 introduces model description and preliminaries. Section 3 gives main results. Section 4 discusses two numerical examples to demonstrate the effectiveness of theoretical results. Finally, Section 5 concludes the paper with some remarks.

\section{Model Description and Preliminaries}

Consider a general class of memristive regulatory-type networks described by the following delay differential equations: for $i=1,2, \ldots, N, j=1,2, \ldots, M$,

$$
\begin{aligned}
& \dot{p}_{i}(t)=-a_{i} p_{i}(t)+\sum_{j=1}^{M} b_{i j}\left(q_{j}(t)\right) f_{j}\left(q_{j}\left(t-\rho_{i j}\right)\right), \\
& \dot{q}_{j}(t)=-c_{j} q_{j}(t)+\sum_{i=1}^{N} d_{j i} p_{i}\left(t-\varrho_{j i}\right)
\end{aligned}
$$

where $p_{i}(t)$ and $q_{j}(t)$ represent the concentration variations of memristive messenger gene $i$ and affiliated organic compound $j$, respectively, $a_{i}>0$ and $c_{j}>0$ denote the degradation rates of memristive messenger gene $i$ and affiliated organic compound $j$, respectively, $d_{j i} \geq 0$ represents the translating rate, nonlinear function $f_{j}(\cdot)$ is bounded and $f_{j}(0)=0,0 \leq \rho_{i j} \leq \tau$ and $0 \leq \varrho_{j i} \leq \tau(\tau \geq 0$ is a constant) denote the regulating delay and the translating delay, respectively, and $b_{i j}\left(q_{j}(t)\right)$ represents regulatory relationship of the network, which is defined as

$$
b_{i j}\left(q_{j}(t)\right)= \begin{cases}\hat{b}_{i j}, & q_{j}(t)<0, \\ \check{b}_{i j}, & q_{j}(t)>0,\end{cases}
$$

where $\widehat{b}_{i j}$ and $\check{b}_{i j}$ are constants.

The initial conditions of system (1) are assumed to be

$$
\begin{aligned}
& p_{i}(s)=\phi_{i}(s), \\
& \begin{array}{l}
q_{j}(s)=\varphi_{j}(s), \\
\quad s \in[-\tau, 0], i=1,2, \ldots, N, j=1,2, \ldots, M,
\end{array}
\end{aligned}
$$

where $\phi_{i}(s)$ and $\varphi_{j}(s)$ are both continuous functions defined on $[-\tau, 0]$.
In addition, we also assume that the nonlinear function $f_{j}(\cdot)(j=1,2, \ldots, M)$ satisfies the Lipschitz condition with the Lipschitz constant $l_{j}>0$; that is,

$$
\begin{aligned}
\left|f_{j}(\chi)-f_{j}(\hat{\chi})\right| \leq l_{j}|\chi-\hat{\chi}| & \\
& j=1,2, \ldots, M, \forall \chi, \hat{\chi} \in \mathfrak{R} .
\end{aligned}
$$

In this paper, solutions of all the systems considered in the following are in Filippov's sense. $K(\mathscr{P})$ denotes closure of the convex hull of set $\mathscr{P} . \operatorname{co}\{\widetilde{\Pi}, \widehat{\Pi}\}$ denotes closure of the convex hull generated by real numbers $\widetilde{\Pi}$ and $\widehat{\Pi}$. Let $\bar{b}_{i j}=$ $\max \left\{\widehat{b}_{i j}, \check{b}_{i j}\right\}, \underline{b}_{i j}=\min \left\{\widehat{b}_{i j}, \check{b}_{i j}\right\}$, and $\widetilde{b}_{i j}=\max \left\{\left|\widehat{b}_{i j}\right|,\left|\check{b}_{i j}\right|\right\}$, for $i=1,2, \ldots, N, j=1,2, \ldots, M$.

When considering memristive regulatory-type networks (1), throughout this paper, let us define the set-valued maps as follows:

$$
K\left(b_{i j}\left(q_{j}(t)\right)\right)= \begin{cases}\widehat{b}_{i j}, & q_{j}(t)<0, \\ \operatorname{co}\left\{\widehat{b}_{i j}, \check{b}_{i j}\right\}, & q_{j}(t)=0, \\ \check{b}_{i j}, & q_{j}(t)>0 .\end{cases}
$$

Obviously, for $i=1,2, \ldots, N, j=1,2, \ldots, M$,

$$
\operatorname{co}\left\{\widehat{b}_{i j}, \check{b}_{i j}\right\}=\left[\underline{b}_{i j}, \bar{b}_{i j}\right] \text {. }
$$

By the theory of differential inclusions, from (1), then for $i=1,2, \ldots, N, j=1,2, \ldots, M$,

$$
\begin{aligned}
& \dot{p}_{i}(t) \in-a_{i} p_{i}(t)+\sum_{j=1}^{M} K\left(b_{i j}\left(q_{j}(t)\right)\right) f_{j}\left(q_{j}\left(t-\rho_{i j}\right)\right), \\
& \dot{q}_{j}(t)=-c_{j} q_{j}(t)+\sum_{i=1}^{N} d_{j i} p_{i}\left(t-\varrho_{j i}\right) .
\end{aligned}
$$

A solution $p(t)=\left(p_{1}(t), p_{2}(t), \ldots, p_{N}(t)\right)^{T}, q(t)=\left(q_{1}(t)\right.$, $\left.q_{2}(t), \ldots, q_{M}(t)\right)^{T}$ in the sense of Filippov of system (1) with initial conditions $p_{i}(s)=\phi_{i}(s), q_{j}(s)=\varphi_{j}(s)$, and $s \in[-\tau, 0]$, $i=1,2, \ldots, N, j=1,2, \ldots, M$, is absolutely continuous on any compact interval of $[0,+\infty)$, and

$$
\begin{aligned}
& \dot{p}_{i}(t) \in-a_{i} p_{i}(t)+\sum_{j=1}^{M} K\left(b_{i j}\left(q_{j}(t)\right)\right) f_{j}\left(q_{j}\left(t-\rho_{i j}\right)\right), \\
& \dot{q}_{j}(t)=-c_{j} q_{j}(t)+\sum_{i=1}^{N} d_{j i} p_{i}\left(t-\varrho_{j i}\right) .
\end{aligned}
$$

Definition 1. The constant vectors $p^{*}=\left(p_{1}^{*}, p_{2}^{*}, \ldots, p_{N}^{*}\right)^{T}$ and $q^{*}=\left(q_{1}^{*}, q_{2}^{*}, \ldots, q_{M}^{*}\right)^{T}$ are called an equilibrium point of system (1), if for $i=1,2, \ldots, N, j=1,2, \ldots, M$,

$$
\begin{aligned}
& 0 \in-a_{i} p_{i}^{*}+\sum_{j=1}^{M} K\left(b_{i j}\left(q_{j}^{*}\right)\right) f_{j}\left(q_{j}^{*}\right), \\
& 0=-c_{j} q_{j}^{*}+\sum_{i=1}^{N} d_{j i} p_{i}^{*} .
\end{aligned}
$$


Definition 2. The equilibrium point of system (1) is said to be globally asymptotically stable if it is locally stable in sense of Lyapunov and globally attractive.

According to Lyapunov direct method, from Definition 2, as we know, if there exists an appropriate Lyapunov function $V$ which is positive definite and radially unbounded, such that the time-derivative of $V$ along the trajectory of system (1) is negative definite, then the equilibrium point of system (1) is globally asymptotically stable.

Definition 3. For drive system $\dot{X}(t)=\mathscr{F}(t, \mathscr{X}), \mathscr{X} \in \mathfrak{R}^{n}$, response system $\dot{\mathcal{Y}}(t)=\mathscr{G}(t, \mathscr{Y}, U), \mathscr{Y} \in \mathfrak{R}^{n}$, and $U \in \mathfrak{R}^{n}$, define the synchronization error signal $\mathscr{E}(t)=\mathscr{X}(t)-\mathscr{Y}(t)$, $\mathscr{E} \in \mathfrak{R}^{n}$; then the error dynamics can be expressed by the following form:

$$
\dot{\mathscr{E}}(t)=\mathscr{F}(t, \mathscr{X})-\mathscr{G}(t, \mathscr{Y}, U),
$$

and we say that the response system can be globally asymptotically synchronized with the drive system if the zero solution of error system is globally asymptotically stable.

\section{Main Results}

In this section, we will first give two lemmas, which play important role in the analysis and synthesis of memristive regulatory-type networks (1).

Lemma 4. In system (1) at least one equilibrium point exists: $p^{*}=\left(p_{1}^{*}, p_{2}^{*}, \ldots, p_{N}^{*}\right)^{T} ; q^{*}=\left(q_{1}^{*}, q_{2}^{*}, \ldots, q_{M}^{*}\right)^{T}$.

Lemma 5. For system (1), we have

$$
\begin{aligned}
& \left|K\left(b_{i j}\left(\mathscr{X}_{j}\right)\right) f_{j}\left(\mathscr{X}_{j}\right)-K\left(b_{i j}\left(\mathscr{Y}_{j}\right)\right) f_{j}\left(\mathscr{Y}_{j}\right)\right| \\
& \leq \widetilde{b}_{i j} l_{j}\left|\mathscr{X}_{j}-\mathscr{Y}_{j}\right|, \\
& \quad \forall i=1,2, \ldots, N, j=1,2, \ldots, M, \forall \mathscr{X}_{j}, \mathscr{Y}_{j} \in \mathfrak{R},
\end{aligned}
$$

where $K\left(b_{i j}\left(\mathscr{X}_{j}\right)\right)$ and $K\left(b_{i j}\left(\mathscr{Y}_{j}\right)\right)$ are defined as those in (5).

Using standard arguments as Lemmas 1 and 2 in [15], Lemmas 4 and 5 of this paper can be proved, respectively.

3.1. Global Asymptotic Stability. According to Lemma 4, memristive regulatory-type networks (1) have the equilibrium points $p^{*}=\left(p_{1}^{*}, p_{2}^{*}, \ldots, p_{N}^{*}\right)^{T}$ and $q^{*}=\left(q_{1}^{*}, q_{2}^{*}, \ldots, q_{M}^{*}\right)^{T}$; we shift the equilibrium points $p^{*}=\left(p_{1}^{*}, p_{2}^{*}, \ldots, p_{N}^{*}\right)^{T}$ and $q^{*}=\left(q_{1}^{*}, q_{2}^{*}, \ldots, q_{M}^{*}\right)^{T}$ to the origin by the translation $x_{i}(t)=$ $p_{i}(t)-p_{i}^{*}$ and $y_{j}(t)=q_{j}(t)-q_{j}^{*}$ in the differential inclusion (7), which results in

$$
\begin{aligned}
& \dot{x}_{i}(t) \in-a_{i} x_{i}(t) \\
&+\sum_{j=1}^{M} K\left(b_{i j}\left(y_{j}(t)\right)\right) f_{j}\left(y_{j}\left(t-\rho_{i j}\right)\right), \\
& \dot{y}_{j}(t)=-c_{j} y_{j}(t)+\sum_{i=1}^{N} d_{j i} x_{i}\left(t-\varrho_{j i}\right),
\end{aligned}
$$

where

$$
\begin{aligned}
K( & \left.b_{i j}\left(y_{j}(t)\right)\right) f_{j}\left(y_{j}\left(t-\rho_{i j}\right)\right) \\
= & K\left(b_{i j}\left(y_{j}(t)+q_{j}^{*}\right)\right) f_{j}\left(y_{j}\left(t-\rho_{i j}\right)+q_{j}^{*}\right) \\
& \quad-K\left(b_{i j}\left(q_{j}^{*}\right)\right) f_{j}\left(q_{j}^{*}\right) .
\end{aligned}
$$

According to Lemma 5,

$$
\begin{gathered}
\left|K\left(b_{i j}\left(y_{j}(t)\right)\right) f_{j}\left(y_{j}\left(t-\rho_{i j}\right)\right)\right| \leq \widetilde{b}_{i j} l_{j}\left|y_{j}\left(t-\rho_{i j}\right)\right| . \\
\text { From (12)-(14), for } i=1,2, \ldots, N, j=1,2, \ldots, M, \\
\left|\dot{x}_{i}(t)\right| \leq-a_{i}\left|x_{i}(t)\right|+\sum_{j=1}^{M} \widetilde{b}_{i j} l_{j}\left|y_{j}\left(t-\rho_{i j}\right)\right|, \\
\left|\dot{y}_{j}(t)\right|=-c_{j}\left|y_{j}(t)\right|+\sum_{i=1}^{N} d_{j i}\left|x_{i}\left(t-\varrho_{j i}\right)\right| .
\end{gathered}
$$

Theorem 6. The equilibrium points $p^{*}=\left(p_{1}^{*}, p_{2}^{*}, \ldots, p_{N}^{*}\right)^{T}$ and $q^{*}=\left(q_{1}^{*}, q_{2}^{*}, \ldots, q_{M}^{*}\right)^{T}$ of system (1) is globally asymptotically stable, if the following matrix

$$
\mathscr{W}=\left(\begin{array}{cc}
\mathscr{A} & -\mathscr{B} \\
-\mathscr{D} & \mathscr{C}
\end{array}\right)_{(N+M) \times(N+M)}
$$

is a nonsingular $M$-matrix, where $\mathscr{A}=\operatorname{diag}\left(a_{1}, a_{2}, \ldots, a_{N}\right)$, $\mathscr{B}=\left(\tilde{b}_{i j} l_{j}\right)_{N \times M}, \mathscr{C}=\operatorname{diag}\left(c_{1}, c_{2}, \ldots, c_{M}\right)$, and $\mathscr{D}=\left(d_{j i}\right)_{M \times N}$.

Proof. Since matrix $\mathscr{W}$ is a nonsingular $M$-matrix, by the $M$ matrix theory, it follows that $\mathscr{W}^{T}$ is a nonsingular $M$-matrix. Based on the fact that $\mathscr{W}^{T}$ is a nonsingular $M$-matrix, then there exists an $(N+M)$-dimensional vector $\eta>0$ such that $\mathscr{W}^{T} \eta>0$; that is,

$$
\begin{gathered}
a_{i} \eta_{i}-\sum_{j=1}^{M} d_{j i} \eta_{N+j}>0, \quad \text { for } i=1,2, \ldots, N, \\
c_{j} \eta_{N+j}-\sum_{i=1}^{N} \tilde{b}_{i j} l_{j} \eta_{i}>0, \quad \text { for } j=1,2, \ldots, M .
\end{gathered}
$$

Choose

$$
\begin{aligned}
& \vartheta_{1}=\min _{1 \leq i \leq N}\left\{a_{i} \eta_{i}-\sum_{j=1}^{M} d_{j i} \eta_{N+j}\right\}, \\
& \vartheta_{2}=\min _{1 \leq j \leq M}\left\{c_{j} \eta_{N+j}-\sum_{i=1}^{N} \widetilde{b}_{i j} l_{j} \eta_{i}\right\},
\end{aligned}
$$

and then we get

$$
\vartheta=\min \left\{\vartheta_{1}, \vartheta_{2}\right\}>0 .
$$


Consider the following positive definite and radially unbounded Lyapunov function:

$$
\begin{aligned}
V(x(t), y(t))= & \sum_{i=1}^{N} \eta_{i}\left|x_{i}(t)\right|+\sum_{j=1}^{M} \eta_{N+j}\left|y_{j}(t)\right| \\
& +\sum_{j=1}^{M} \eta_{N+j} \sum_{i=1}^{N} d_{j i} \int_{t-\varrho_{j i}}^{t}\left|x_{i}(s)\right| \mathrm{d} s \\
& +\sum_{i=1}^{N} \eta_{i} \sum_{j=1}^{M} \widetilde{b}_{i j} l_{j} \int_{t-\rho_{i j}}^{t}\left|y_{j}(s)\right| \mathrm{d} s .
\end{aligned}
$$

Calculating the upper right Dini derivative of $V(x(t)$, $y(t))$ along the trajectory of system (12) yields

$$
\begin{aligned}
& D^{+} V(x(t), y(t)) \\
& \leq \sum_{i=1}^{N} \eta_{i}\left[-a_{i}\left|x_{i}(t)\right|+\sum_{j=1}^{M} \widetilde{b}_{i j} l_{j}\left|y_{j}\left(t-\rho_{i j}\right)\right|\right] \\
& +\sum_{j=1}^{M} \eta_{N+j}\left[-c_{j}\left|y_{j}(t)\right|+\sum_{i=1}^{N} d_{j i}\left|x_{i}\left(t-\varrho_{j i}\right)\right|\right] \\
& +\sum_{j=1}^{M} \eta_{N+j} \sum_{i=1}^{N} d_{j i}\left[\left|x_{i}(t)\right|-\left|x_{i}\left(t-\varrho_{j i}\right)\right|\right] \\
& +\sum_{i=1}^{N} \eta_{i} \sum_{j=1}^{M} \widetilde{b}_{i j} l_{j}\left[\left|y_{j}(t)\right|-\left|y_{j}\left(t-\rho_{i j}\right)\right|\right] \\
& =-\sum_{i=1}^{N} \eta_{i} a_{i}\left|x_{i}(t)\right|-\sum_{j=1}^{M} \eta_{N+j} c_{j}\left|y_{j}(t)\right| \\
& +\sum_{j=1}^{M} \eta_{N+j} \sum_{i=1}^{N} d_{j i}\left|x_{i}(t)\right|+\sum_{i=1}^{N} \eta_{i} \sum_{j=1}^{M} \widetilde{b}_{i j} l_{j}\left|y_{j}(t)\right| \\
& =\sum_{i=1}^{N}\left[-\eta_{i} a_{\mathrm{i}}+\sum_{j=1}^{M} \eta_{N+j} d_{j i}\right]\left|x_{i}(t)\right| \\
& +\sum_{j=1}^{M}\left[-\eta_{N+j} c_{j}+\sum_{i=1}^{N} \eta_{i} \widetilde{b}_{i j} l_{j}\right]\left|y_{j}(t)\right| \\
& \leq-\vartheta\left[\sum_{i=1}^{N}\left|x_{i}(t)\right|+\sum_{j=1}^{M}\left|y_{j}(t)\right|\right] \text {. }
\end{aligned}
$$

By Lyapunov global asymptotic stability theory, we can conclude system (12) is globally asymptotically stable. Thus, the equilibrium points $p^{*}=\left(p_{1}^{*}, p_{2}^{*}, \ldots, p_{N}^{*}\right)^{T}$ and $q^{*}=$ $\left(q_{1}^{*}, q_{2}^{*}, \ldots, q_{M}^{*}\right)^{T}$ of system (1) are globally asymptotically stable. The proof is completed.
Next we extend Theorem 6 to other possible cases.

Corollary 7. The equilibrium points $p^{*}=\left(p_{1}^{*}, p_{2}^{*}, \ldots, p_{N}^{*}\right)^{T}$ and $q^{*}=\left(q_{1}^{*}, q_{2}^{*}, \ldots, q_{M}^{*}\right)^{T}$ of system (1) are globally asymptotically stable, if

$$
\begin{aligned}
& a_{i}>\sum_{j=1}^{M} d_{j i}, \quad \text { for } i=1,2, \ldots, N, \\
& c_{j}>\sum_{i=1}^{N} \widetilde{b}_{i j} l_{j}, \quad \text { for } j=1,2, \ldots, M .
\end{aligned}
$$

Proof. Select the $(N+M)$-dimensional unit vector as $\eta$ in the proof of Theorem 6, from (22); it follows that (17) hold. Therefore, the conclusion of Corollary 7 is obvious.

Corollary 8. When $M=N$, the equilibrium points $p^{*}=$ $\left(p_{1}^{*}, p_{2}^{*}, \ldots, p_{N}^{*}\right)^{T}$ and $q^{*}=\left(q_{1}^{*}, q_{2}^{*}, \ldots, q_{M}^{*}\right)^{T}$ of system (1) are globally asymptotically stable, if the matrix

$$
\mathscr{W}=\mathscr{A} \mathscr{C}-\mathscr{D} \mathscr{B}
$$

is a nonsingular $M$-matrix, where $\mathscr{A}=\operatorname{diag}\left(a_{1}, a_{2}, \ldots, a_{N}\right)$, $\mathscr{B}=\left(\widetilde{b}_{i j} l_{j}\right)_{N \times M}, \mathscr{C}=\operatorname{diag}\left(c_{1}, c_{2}, \ldots, c_{M}\right)$, and $\mathscr{D}=\left(d_{j i}\right)_{M \times N}$.

Proof. The proof is a direct result of Theorem 6.

3.2. Global Asymptotic Synchronization. Let (1) be the drive memristive regulatory-type networks. The response memristive regulatory-type networks are described by the following: for $i=1,2, \ldots, N, j=1,2, \ldots, M$,

$$
\begin{aligned}
\dot{\mathscr{P}}_{i}(t)= & -a_{i} \mathscr{P}_{i}(t)+\sum_{j=1}^{M} b_{i j}\left(\mathscr{Q}_{j}(t)\right) f_{j}\left(\mathscr{Q}_{j}\left(t-\rho_{i j}\right)\right) \\
& +\mathscr{U}_{i}(t), \\
\dot{Q}_{j}(t)= & -c_{j} \mathscr{Q}_{j}(t)+\sum_{i=1}^{N} d_{j i} \mathscr{P}_{i}\left(t-\varrho_{j i}\right)+\mathscr{V}_{j}(t),
\end{aligned}
$$

where $\mathscr{U}_{i}(t), \mathscr{V}_{j}(t), i=1,2, \ldots, N, j=1,2, \ldots, M$, denote the appropriate control inputs that will be designed in order to obtain a certain control objective.

Next, the linear feedback scheme is used to achieve synchronization between drive memristive regulatory-type networks (1) and response memristive regulatory-type networks (24); that is, the controllers $\mathscr{U}_{i}(t), \mathscr{V}_{j}(t), i=1,2, \ldots, N$, $j=1,2, \ldots, M$, are designed as follows:

$$
\begin{aligned}
& \mathscr{U}_{i}(t)=\mathscr{K}_{i}\left(p_{i}(t)-\mathscr{P}_{i}(t)\right), \\
& \mathscr{V}_{j}(t)=\mathscr{H}_{j}\left(q_{j}(t)-\mathscr{Q}_{j}(t)\right),
\end{aligned}
$$

where $\mathscr{K}_{i}>0, \mathscr{H}_{j}>0$ denote the control gains.

Let

$$
\begin{aligned}
e_{i}(t) & =p_{i}(t)-\mathscr{P}_{i}(t), \\
\mathscr{E}_{j}(t) & =q_{j}(t)-\mathscr{Q}_{j}(t),
\end{aligned}
$$


for $i=1,2, \ldots, N, j=1,2, \ldots, M$. Then by drive memristive regulatory-type networks (1), response memristive regulatory-type networks (24), and the controllers (25), the error system can be described by

$$
\begin{gathered}
\dot{e}_{i}(t)=-a_{i} e_{i}(t)+\left[\sum_{j=1}^{M} b_{i j}\left(q_{j}(t)\right) f_{j}\left(q_{j}\left(t-\rho_{i j}\right)\right)\right. \\
\left.-\sum_{j=1}^{M} b_{i j}\left(\mathscr{Q}_{j}(t)\right) f_{j}\left(\mathscr{Q}_{j}\left(t-\rho_{i j}\right)\right)\right]-\mathscr{K}_{i} e_{i}(t), \\
\dot{\mathscr{E}}_{j}(t)=-c_{j} \mathscr{E}_{j}(t)+\sum_{i=1}^{N} d_{j i} e_{i}\left(t-\varrho_{j i}\right)-\mathscr{H}_{j} \mathscr{E}_{j}(t),
\end{gathered}
$$

for $i=1,2, \ldots, N, j=1,2, \ldots, M$.

To apply the theories of set-valued maps and differential inclusions, (27) is equivalent to

$$
\begin{aligned}
\dot{e}_{i}(t) & \in-a_{i} e_{i}(t) \\
+ & {\left[\sum_{j=1}^{M} K\left(b_{i j}\left(q_{j}(t)\right)\right) f_{j}\left(q_{j}\left(t-\rho_{i j}\right)\right)\right.} \\
& \left.-\sum_{j=1}^{M} K\left(b_{i j}\left(Q_{j}(t)\right)\right) f_{j}\left(Q_{j}\left(t-\rho_{i j}\right)\right)\right]-\mathscr{K}_{i} e_{i}(t), \\
\dot{\mathscr{E}}_{j}(t) & =-c_{j} \mathscr{E}_{j}(t)+\sum_{i=1}^{N} d_{j i} e_{i}\left(t-\varrho_{j i}\right)-\mathscr{H}_{j} \mathscr{E}_{j}(t) .
\end{aligned}
$$

According to Lemma 5,

$$
\begin{aligned}
& \mid K\left(b_{i j}\left(q_{j}(t)\right)\right) f_{j}\left(q_{j}\left(t-\rho_{i j}\right)\right) \\
& \quad-K\left(b_{i j}\left(Q_{j}(t)\right)\right) f_{j}\left(Q_{j}\left(t-\rho_{i j}\right)\right) \mid \\
& \quad \leq \widetilde{b}_{i j} l_{j}\left|\mathscr{E}_{j}\left(t-\rho_{i j}\right)\right| .
\end{aligned}
$$

From (28) and (29), for $i=1,2, \ldots, N, j=1,2, \ldots, M$,

$$
\begin{array}{r}
\left|\dot{e}_{i}(t)\right| \leq-\left(a_{i}+\mathscr{K}_{i}\right)\left|e_{i}(t)\right|+\sum_{j=1}^{M} \widetilde{b}_{i j} l_{j}\left|\mathscr{E}_{j}\left(t-\rho_{i j}\right)\right|, \\
\left|\dot{\mathscr{E}}_{j}(t)\right|=-\left(c_{j}+\mathscr{H}_{j}\right)\left|\mathscr{E}_{j}(t)\right|+\sum_{i=1}^{N} d_{j i}\left|e_{i}\left(t-\varrho_{j i}\right)\right| .
\end{array}
$$

Theorem 9. The zero solution of system (28) is globally asymptotically stable; that is, the response system (24) can be globally asymptotically synchronized with the drive system (1), if the following matrix

$$
\mathscr{W}=\left(\begin{array}{cc}
\mathscr{A} & -\mathscr{B} \\
-\mathscr{D} & \mathscr{C}
\end{array}\right)_{(N+M) \times(N+M)}
$$

is a nonsingular $M$-matrix, where $\mathscr{A}=\operatorname{diag}\left(a_{1}+\mathscr{K}_{1}, a_{2}+\right.$ $\left.\mathscr{K}_{2}, \ldots, a_{N}+\mathscr{K}_{N}\right), \mathscr{B}=\left(\widetilde{b}_{i j} l_{j}\right)_{N \times M}, \mathscr{C}=\operatorname{diag}\left(c_{1}+\mathscr{H}_{1}, c_{2}+\right.$ $\left.\mathscr{H}_{2}, \ldots, c_{M}+\mathscr{H}_{M}\right)$, and $\mathscr{D}=\left(d_{j i}\right)_{M \times N}$.
Proof. Since matrix $\mathscr{W}$ is a nonsingular $M$-matrix, by the $M$ matrix theory, it follows that $\mathscr{W}^{T}$ is a nonsingular $M$-matrix. Based on the fact that $\mathscr{W}^{T}$ is a nonsingular $M$-matrix, then there exists an $(N+M)$-dimensional vector $\eta>0$ such that $\mathscr{W}^{T} \eta>0$; that is,

$$
\begin{gathered}
\left(a_{i}+\mathscr{K}_{i}\right) \eta_{i}-\sum_{j=1}^{M} d_{j i} \eta_{N+j}>0, \quad \text { for } i=1,2, \ldots, N, \\
\left(c_{j}+\mathscr{H}_{j}\right) \eta_{N+j}-\sum_{i=1}^{N} \widetilde{b}_{i j} l_{j} \eta_{i}>0, \quad \text { for } j=1,2, \ldots, M .
\end{gathered}
$$

Choose

$$
\begin{aligned}
& \vartheta_{1}=\min _{1 \leq i \leq N}\left\{\left(a_{i}+\mathscr{K}_{i}\right) \eta_{i}-\sum_{j=1}^{M} d_{j i} \eta_{N+j}\right\}, \\
& \vartheta_{2}=\min _{1 \leq j \leq M}\left\{\left(c_{j}+\mathscr{H}_{j}\right) \eta_{N+j}-\sum_{i=1}^{N} \widetilde{b}_{i j} l_{j} \eta_{i}\right\},
\end{aligned}
$$

and then we get

$$
\vartheta=\min \left\{\vartheta_{1}, \vartheta_{2}\right\}>0 .
$$

Consider the following positive definite and radially unbounded Lyapunov function:

$$
\begin{aligned}
V(e(t), \mathscr{E}(t))= & \sum_{i=1}^{N} \eta_{i}\left|e_{i}(t)\right|+\sum_{j=1}^{M} \eta_{N+j}\left|\mathscr{E}_{j}(t)\right| \\
& +\sum_{j=1}^{M} \eta_{N+j} \sum_{i=1}^{N} d_{j i} \int_{t-\varrho_{j i}}^{t}\left|e_{i}(s)\right| \mathrm{d} s \\
& +\sum_{i=1}^{N} \eta_{i} \sum_{j=1}^{M} \widetilde{b}_{i j} l_{j} \int_{t-\rho_{i j}}^{t}\left|\mathscr{E}_{j}(s)\right| \mathrm{d} s .
\end{aligned}
$$

Calculating the upper right Dini derivative of $V(e(t)$, $\mathscr{E}(t))$ along the trajectory of system (28) yields

$$
\begin{aligned}
& D^{+} V(e(t), \mathscr{E}(t)) \leq \sum_{i=1}^{N} \eta_{i}\left[-\left(a_{i}+\mathscr{K}_{i}\right)\left|e_{i}(t)\right|\right. \\
& \left.+\sum_{j=1}^{M} \widetilde{b}_{i j} l_{j}\left|\mathscr{E}_{j}\left(t-\rho_{i j}\right)\right|\right] \\
& +\sum_{j=1}^{M} \eta_{N+j}\left[-\left(c_{j}+\mathscr{H}_{j}\right)\left|\mathscr{E}_{j}(t)\right|\right. \\
& \left.+\sum_{i=1}^{N} d_{j i}\left|e_{i}\left(t-\varrho_{j i}\right)\right|\right]+\sum_{j=1}^{M} \eta_{N+j} \sum_{i=1}^{N} d_{j i}\left[\left|e_{i}(t)\right|\right. \\
& \left.\quad-\left|e_{i}\left(t-\varrho_{j i}\right)\right|\right]+\sum_{i=1}^{N} \eta_{i} \sum_{j=1}^{M} \widetilde{b}_{i j} l_{j}\left[\left|\mathscr{E}_{j}(t)\right|\right. \\
& \left.\quad-\left|\mathscr{E}_{j}\left(t-\rho_{i j}\right)\right|\right]=-\sum_{i=1}^{N} \eta_{i}\left(a_{i}+\mathscr{K}_{i}\right)\left|e_{i}(t)\right|
\end{aligned}
$$




$$
\begin{aligned}
& -\sum_{j=1}^{M} \eta_{N+j}\left(c_{j}+\mathscr{H}_{j}\right)\left|\mathscr{E}_{j}(t)\right|+\sum_{j=1}^{M} \eta_{N+j} \sum_{i=1}^{N} d_{j i}\left|e_{i}(t)\right| \\
& +\sum_{i=1}^{N} \eta_{i} \sum_{j=1}^{M} \widetilde{b}_{i j} l_{j}\left|\mathscr{E}_{j}(t)\right|=\sum_{i=1}^{N}\left[-\eta_{i}\left(a_{i}+\mathscr{K}_{i}\right)\right. \\
& \left.+\sum_{j=1}^{M} \eta_{N+j} d_{j i}\right]\left|e_{i}(t)\right|+\sum_{j=1}^{M}\left[-\eta_{N+j}\left(c_{j}+\mathscr{H}_{j}\right)\right. \\
& \left.+\sum_{i=1}^{N} \eta_{i} \widetilde{b}_{i j} l_{j}\right]\left|\mathscr{E}_{j}(t)\right| \leq-\vartheta\left[\sum_{i=1}^{N}\left|e_{i}(t)\right|\right. \\
& \left.+\sum_{j=1}^{M}\left|\mathscr{E}_{j}(t)\right|\right]
\end{aligned}
$$

By Lyapunov global asymptotic stability theory, we can conclude that system (28) is globally asymptotically stable. Thus, the response system (24) can be globally asymptotically synchronized with the drive system (1). The proof is completed.

Next we extend Theorem 9 to other possible cases.

Corollary 10. The zero solution of system (28) is globally asymptotically stable; that is, the response system (24) can be globally asymptotically synchronized with the drive system (1), if

$$
\begin{aligned}
& a_{i}+\mathscr{K}_{i}>\sum_{j=1}^{M} d_{j i}, \quad \text { for } i=1,2, \ldots, N, \\
& c_{j}+\mathscr{H}_{j}>\sum_{i=1}^{N} \widetilde{b}_{i j} l_{j}, \quad \text { for } j=1,2, \ldots, M .
\end{aligned}
$$

Proof. Select the $(N+M)$-dimensional unit vector as $\eta$ in the proof of Theorem 9, from (37), it follows that (32) hold. Therefore, the conclusion of Corollary 10 is obvious.

Corollary 11. When $M=N$, the zero solution of system (28) is globally asymptotically stable; that is, the response system (24) can be globally asymptotically synchronized with the drive system (1), if the matrix

$$
\mathscr{W}=\mathscr{A} \mathscr{C}-\mathscr{D} \mathscr{B}
$$

is a nonsingular $M$-matrix, where $\mathscr{A}=\operatorname{diag}\left(a_{1}+\mathscr{K}_{1}, a_{2}+\right.$ $\left.\mathscr{K}_{2}, \ldots, a_{N}+\mathscr{K}_{N}\right), \mathscr{B}=\left(\widetilde{b}_{i j} l_{j}\right)_{N \times M}, \mathscr{C}=\operatorname{diag}\left(c_{1}+\mathscr{H}_{1}, c_{2}+\right.$ $\left.\mathscr{H}_{2}, \ldots, c_{M}+\mathscr{H}_{M}\right)$, and $\mathscr{D}=\left(d_{j i}\right)_{M \times N}$.

Proof. The proof is a direct result of Theorem 9.

Remark 12. Theorem 9 and Corollaries 10 and 11 show the feasibility of linear feedback scheme for designing a perfect control in memristive regulatory-type networks, and the sufficient conditions only depend on some system parameters, which are easy to be checked.
Remark 13. Compared with many other control strategies, linear feedback scheme is more suitable for implementation in memristive regulatory-type networks. For one thing, transient states are quite prevalent in memristive regulatory-type networks; that is, state-dependent jump abruptly spikes up or down with uncertainty. For another thing, linear feedback scheme itself is relatively cheaper and simpler to operate. It is more reasonable and implementable for linear feedback scheme only carried out at finite gain and bandwidth.

Remark 14. The asymptotic synchronization strategy contains more general synchronization behaviors. Through the node cluster, asymptotic synchronization in each group can achieve complete synchronization.

\section{Illustrative Examples}

In this section, we discuss two numerical examples to illustrate the theoretical results.

Example 1. Consider the following memristive regulatorytype networks:

$$
\begin{aligned}
& \dot{p}_{i}(t)=-p_{i}(t)+\sum_{j=1}^{2} b_{i j}\left(q_{j}(t)\right) f_{j}\left(q_{j}(t-0.1)\right), \\
& \dot{q}_{j}(t)=-q_{j}(t)+\sum_{i=1}^{3} 0.2 p_{i}(t-0.3),
\end{aligned}
$$

where $i=1,2,3, j=1,2, f_{j}(\nu)=(|\nu+1|-|\nu-1|) / 2$,

$$
b_{i j}\left(q_{j}(t)\right)= \begin{cases}0.3, & q_{j}(t)<0, \\ -0.3, & q_{j}(t)>0\end{cases}
$$

$$
i=1,2,3, j=1,2 \text {. }
$$

Obviously, we can calculate that

$$
\begin{aligned}
\mathscr{A} & =\left(\begin{array}{lll}
1 & 0 & 0 \\
0 & 1 & 0 \\
0 & 0 & 1
\end{array}\right)_{3 \times 3}, \\
-\mathscr{B} & =\left(\begin{array}{cc}
-0.3 & -0.3 \\
-0.3 & -0.3 \\
-0.3 & -0.3
\end{array}\right)_{3 \times 2}, \\
\mathscr{C} & =\left(\begin{array}{cc}
1 & 0 \\
0 & 1
\end{array}\right)_{2 \times 2}, \\
-\mathscr{D} & =\left(\begin{array}{rrr}
-0.2 & -0.2 & -0.2 \\
-0.2 & -0.2 & -0.2
\end{array}\right)_{2 \times 3},
\end{aligned}
$$



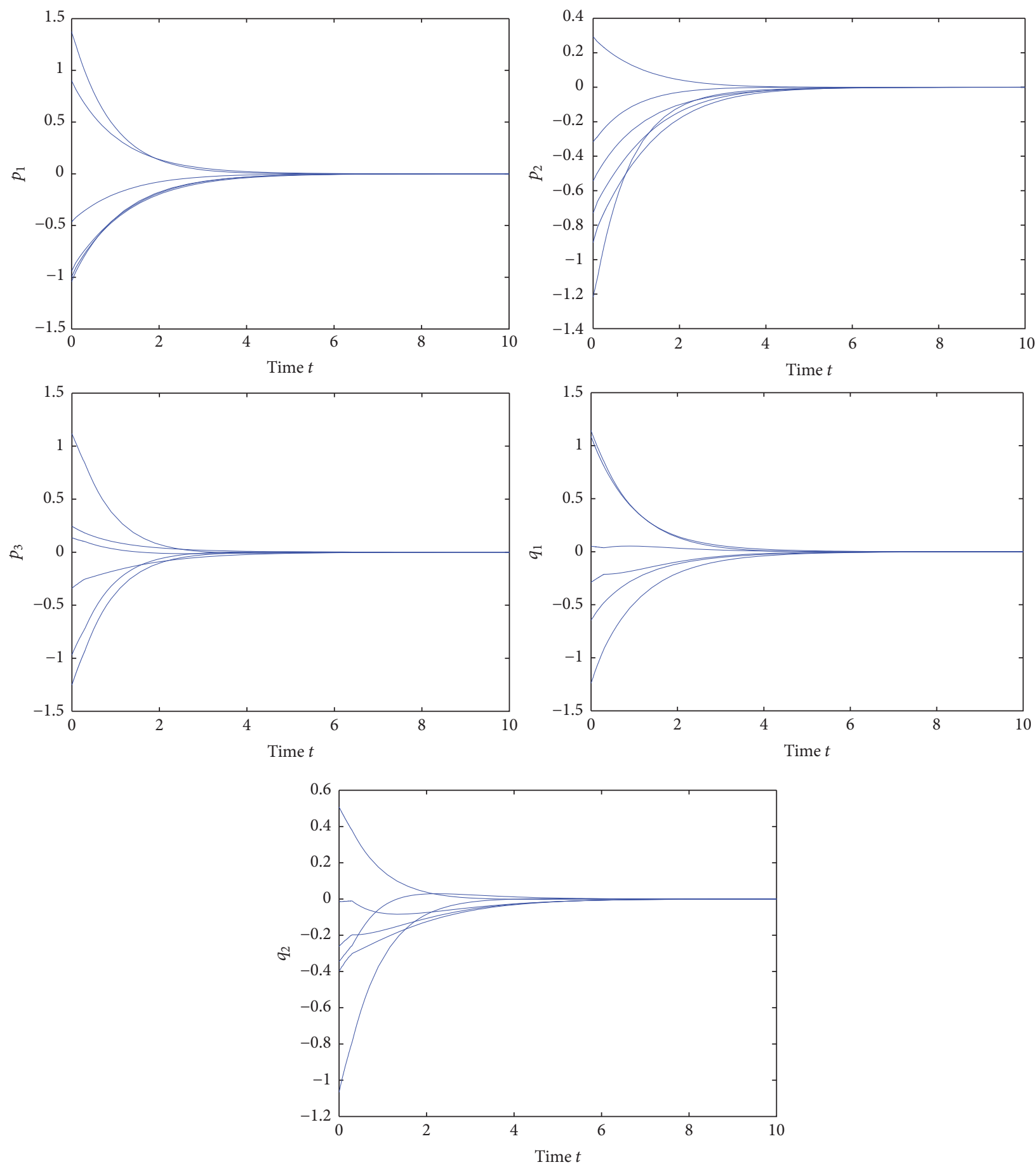

FIgURE 1: Transient behaviors of system (39).

and then

$$
\mathscr{W}=\left(\begin{array}{ccccc}
1 & 0 & 0 & -0.3 & -0.3 \\
0 & 1 & 0 & -0.3 & -0.3 \\
0 & 0 & 1 & -0.3 & -0.3 \\
-0.2 & -0.2 & -0.2 & 1 & 0 \\
-0.2 & -0.2 & -0.2 & 0 & 1
\end{array}\right)_{5 \times 5}
$$

and the eigenvalues of matrix $\mathscr{W}$ are $0.4,1,1,1$, and 1.6; thus the matrix $\mathscr{W}$ is a nonsingular $M$-matrix. According to Theorem 6, system (39) is globally asymptotically stable.

The simulation results of system (39) with some initial values are depicted in Figures 1 and 2. Based on the dynamical evolutions in Figures 1 and 2, we can see that the experimental results agree with the theory very well. 

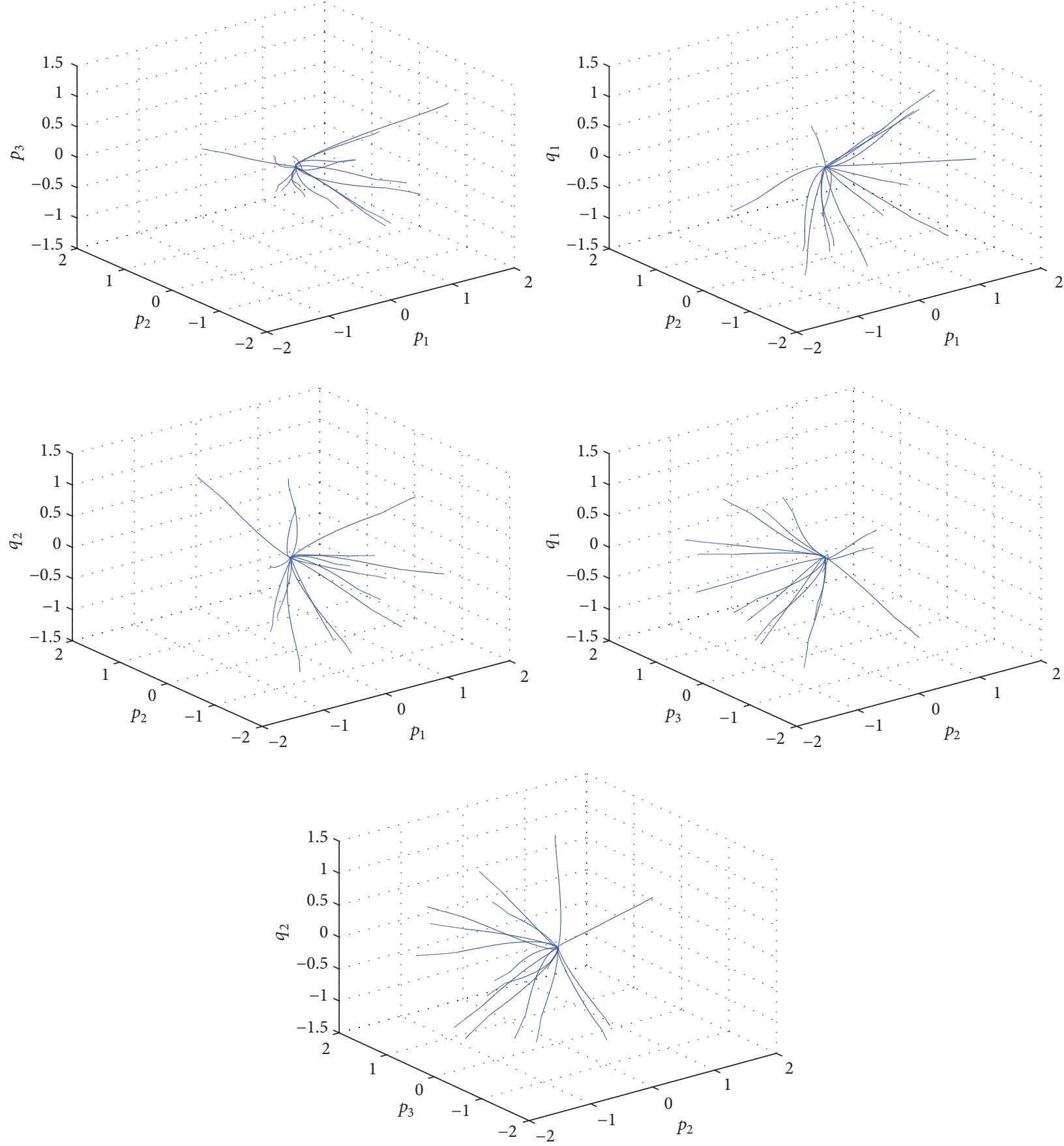

FIGURE 2: Phase portraits of system (39) in the three-dimensional space.

Example 2. Consider the following memristive regulatorytype networks:

$$
\begin{aligned}
& \dot{p}_{i}(t)=-0.2 p_{i}(t)+\sum_{j=1}^{2} b_{i j}\left(q_{j}(t)\right) f_{j}\left(q_{j}(t-0.1)\right), \\
& \dot{q}_{j}(t)=-0.2 q_{j}(t)+\sum_{i=1}^{3} 0.2 p_{i}(t-0.3),
\end{aligned}
$$

where $i=1,2,3, j=1,2, f_{j}(\nu)=(|\nu+1|-|\nu-1|) / 2$,

$$
b_{i j}\left(q_{j}(t)\right)= \begin{cases}0.3, & q_{j}(t)<0, \\ -0.3, & q_{j}(t)>0,\end{cases}
$$

$$
i=1,2,3, j=1,2 \text {. }
$$



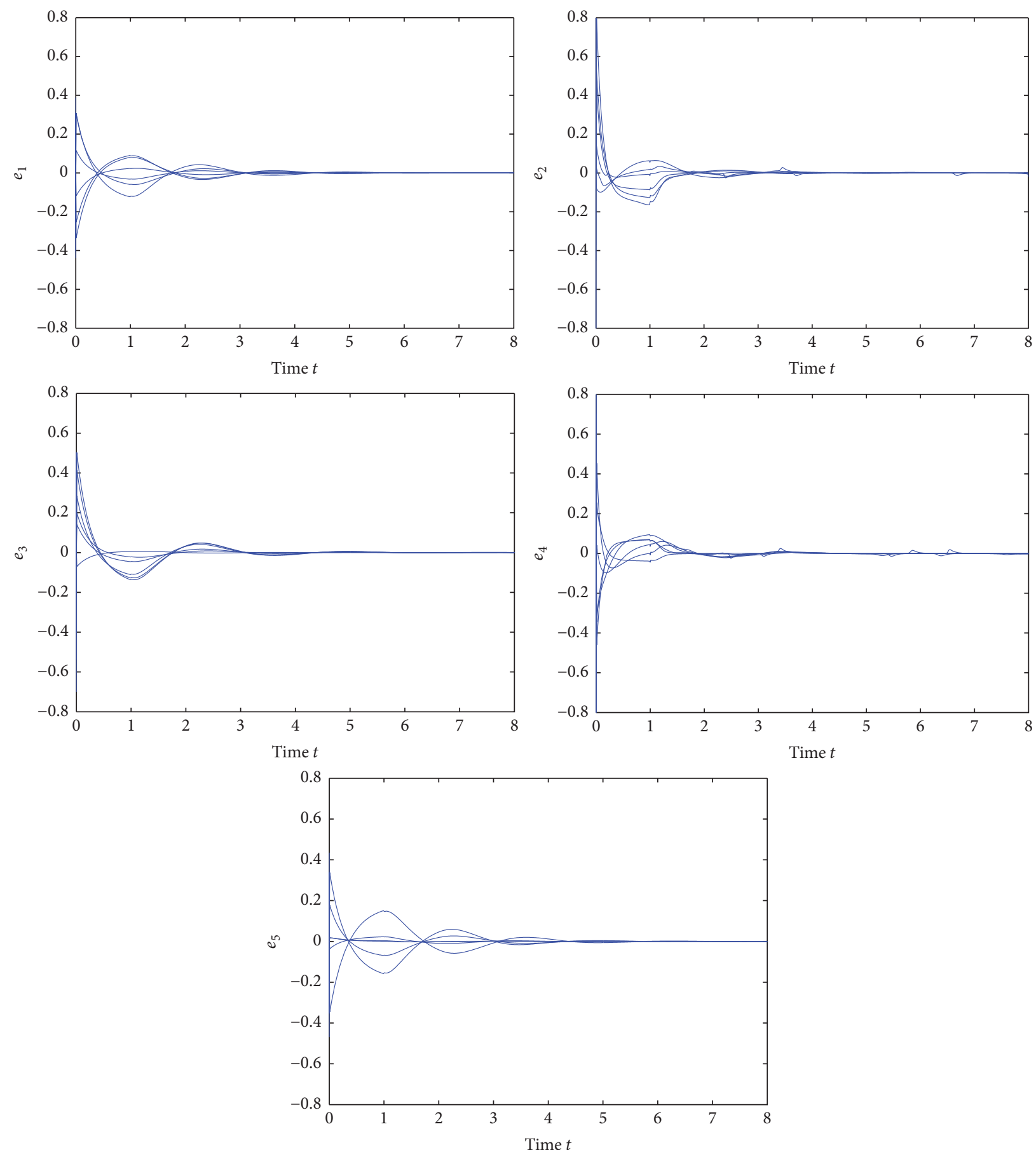

Figure 3: The error dynamics $e_{1}(t)=p_{1}(t)-\mathscr{P}_{1}(t), e_{2}(t)=p_{2}(t)-\mathscr{P}_{2}(t), e_{3}(t)=p_{3}(t)-\mathscr{P}_{3}(t), e_{4}(t)=q_{1}(t)-Q_{1}(t)$, and $e_{5}(t)=q_{2}(t)-Q_{2}(t)$.

Let (43) be the drive system. The response system is described by

$$
\begin{aligned}
\dot{\mathscr{P}}_{i}(t)= & -0.2 \mathscr{P}_{i}(t)+\sum_{j=1}^{2} b_{i j}\left(\mathscr{Q}_{j}(t)\right) f_{j}\left(\mathscr{Q}_{j}(t-0.1)\right) \\
& +\mathscr{U}_{i}(t), \\
\dot{\mathcal{Q}}_{j}(t)= & -0.2 \mathscr{Q}_{j}(t)+\sum_{i=1}^{3} 0.2 \mathscr{P}_{i}(t-0.3)+\mathscr{V}_{j}(t),
\end{aligned}
$$

where $i=1,2,3, j=1,2, f_{j}(\nu)=(|\nu+1|-|\nu-1|) / 2$,

$$
b_{i j}\left(Q_{j}(t)\right)= \begin{cases}0.3, & Q_{j}(t)<0, \\ -0.3, & Q_{j}(t)>0,\end{cases}
$$

The controllers $\mathscr{U}_{i}(t), \mathscr{V}_{j}(t), i=1,2,3, j=1,2$, are designed as follows:

$$
\mathcal{U}_{i}(t)=0.8\left(p_{i}(t)-\mathscr{P}_{i}(t)\right)
$$




$$
\mathscr{V}_{j}(t)=0.8\left(q_{j}(t)-\mathscr{Q}_{j}(t)\right)
$$

and to apply Theorem 9, then we can calculate that

$$
\begin{aligned}
\mathscr{A} & =\left(\begin{array}{lll}
1 & 0 & 0 \\
0 & 1 & 0 \\
0 & 0 & 1
\end{array}\right)_{3 \times 3}, \\
-\mathscr{B} & =\left(\begin{array}{rr}
-0.3 & -0.3 \\
-0.3 & -0.3 \\
-0.3 & -0.3
\end{array}\right)_{3 \times 2}, \\
\mathscr{C} & =\left(\begin{array}{ll}
1 & 0 \\
0 & 1
\end{array}\right)_{2 \times 2}, \\
-\mathscr{D} & =\left(\begin{array}{lll}
-0.2 & -0.2 & -0.2 \\
-0.2 & -0.2 & -0.2
\end{array}\right)_{2 \times 3},
\end{aligned}
$$

and then

$$
\mathscr{W}=\left(\begin{array}{ccccc}
1 & 0 & 0 & -0.3 & -0.3 \\
0 & 1 & 0 & -0.3 & -0.3 \\
0 & 0 & 1 & -0.3 & -0.3 \\
-0.2 & -0.2 & -0.2 & 1 & 0 \\
-0.2 & -0.2 & -0.2 & 0 & 1
\end{array}\right)_{5 \times 5}
$$

and the eigenvalues of matrix $\mathscr{W}$ are $0.4,1,1,1$, and 1.6; thus the matrix $\mathscr{W}$ is a nonsingular $M$-matrix. According to Theorem 9, the response system (45) can be globally asymptotically synchronized with the drive system (43). The simulation result on the error dynamics $e_{1}(t)=p_{1}(t)-\mathscr{P}_{1}(t)$, $e_{2}(t)=p_{2}(t)-\mathscr{P}_{2}(t), e_{3}(t)=p_{3}(t)-\mathscr{P}_{3}(t), e_{4}(t)=q_{1}(t)-$ $\mathbb{Q}_{1}(t)$, and $e_{5}(t)=q_{2}(t)-\mathbb{Q}_{2}(t)$, with some initial values, is depicted in Figure 3. The dynamical evolutions in Figure 3 clearly indicate that the controller designed performs well.

\section{Conclusion}

Memristive network can achieve more expedient goal-finding behavior in spiking networks via memristive connections, which has aroused considerable interest by electronics researchers. The practical applications of memristive network popularizes real-time processing and recognition of natural signals. It is of great significance to investigate its nonlinear dynamics. In this paper, we study global asymptotic stability and global asymptotic synchronization for memristive regulatory-type networks, based on the $M$-matrix theory and Lyapunov stability theory. These criteria, which can be directly derived from the system parameters, are easily verified. The theoretical results developed in this paper may be applied to the synthesis of memristive regulatory-type networks.

\section{Competing Interests}

The author declares that there is no conflict of interests regarding the publication of this paper.

\section{Acknowledgments}

The work is supported by the Research Project of Hubei Provincial Department of Education of China under Grant T201412.

\section{References}

[1] T. Li, S. Duan, J. Liu, L. Wang, and T. Huang, "A spintronic memristor-based neural network with radial basis function for robotic manipulator control implementation," IEEE Transactions on Systems, Man, and Cybernetics: Systems, vol. 46, no. 4, pp. 582-588, 2016.

[2] S. P. Adhikari, H. Kim, R. K. Budhathoki, C. Yang, and L. O. Chua, "A circuit-based learning architecture for multilayer neural networks with memristor bridge synapses," IEEE Transactions on Circuits and Systems I: Regular Papers, vol. 62, no. 1, pp. 215-223, 2015.

[3] Z. Y. Guo, J. Wang, and Z. Yan, "Global exponential dissipativity and stabilization of memristor-based recurrent neural networks with time-varying delays," Neural Networks, vol. 48, pp. 158-172, 2013.

[4] Z. Guo, J. Wang, and Z. Yan, "Attractivity analysis of memristorbased cellular neural networks with time-varying delays," IEEE Transactions on Neural Networks and Learning Systems, vol. 25, no. 4, pp. 704-717, 2014.

[5] Y. V. Pershin and M. Di Ventra, "Experimental demonstration of associative memory with memristive neural networks," Neural Networks, vol. 23, no. 7, pp. 881-886, 2010.

[6] X. Wang, C. Li, and T. Huang, "Delay-dependent robust stability and stabilization of uncertain memristive delay neural networks," Neurocomputing, vol. 140, pp. 155-161, 2014.

[7] X. Wang, C. Li, T. Huang, and S. Duan, "Global exponential stability of a class of memristive neural networks with timevarying delays," Neural Computing and Applications, vol. 24, no. 7-8, pp. 1707-1715, 2014.

[8] X. S. Yang and D. W. C. Ho, "Synchronization of delayed memristive neural networks: robust analysis approach," IEEE Transactions on Cybernetics, vol. 46, no. 12, pp. 3377-3387, 2016.

[9] Z. Wang, S. Ding, Z. Huang, and H. Zhang, "Exponential stability and stabilization of delayed memristive neural networks based on quadratic convex combination method," IEEE Transactions on Neural Networks and Learning Systems, vol. 27, no. 11, pp. 2337-2350, 2016.

[10] A. Wu and Z. Zeng, "Global Mittag-Leffler stabilization of fractional-order memristive neural networks," IEEE Transactions on Neural Networks and Learning Systems, vol. 28, no. 1, pp. 206-217, 2017.

[11] L. M. Wang and Y. Shen, "Finite-time stabilizability and instabilizability of delayed memristive neural networks with nonlinear discontinuous controller," IEEE Transactions on Neural Networks and Learning Systems, vol. 26, no. 11, pp. 2914-2924, 2015.

[12] S. Yang, Z. Guo, and J. Wang, "Robust synchronization of multiple memristive neural networks with uncertain parameters via 
nonlinear coupling," IEEE Transactions on Systems, Man, and Cybernetics: Systems, vol. 45, no. 7, pp. 1077-1086, 2015.

[13] A. Wu and Z. Zhigang, "Lagrange stability of memristive neural networks with discrete and distributed delays," IEEE Transactions on Neural Networks and Learning Systems, vol. 25, no. 4, pp. 690-703, 2014.

[14] D. Querlioz, O. Bichler, P. Dollfus, and C. Gamrat, "Immunity to device variations in a spiking neural network with memristive nanodevices," IEEE Transactions on Nanotechnology, vol. 12, no. 3, pp. 288-295, 2013.

[15] A. Wu and Z. Zeng, "An improved criterion for stability and attractability of memristive neural networks with time-varying delays," Neurocomputing, vol. 145, pp. 316-323, 2014.

[16] M. Al-Shedivat, R. Naous, G. Cauwenberghs, and K. N. Salama, "Memristors empower spiking neurons with stochasticity," IEEE Journal on Emerging and Selected Topics in Circuits and Systems, vol. 5, no. 2, pp. 242-253, 2015.

[17] G. D. Zhang and Y. Shen, "New algebraic criteria for synchronization stability of chaotic memristive neural networks with time-varying delays," IEEE Transactions on Neural Networks and Learning Systems, vol. 24, no. 10, pp. 1701-1707, 2013.

[18] G. Zhang, Y. Shen, Q. Yin, and J. Sun, "Global exponential periodicity and stability of a class of memristor-based recurrent neural networks with multiple delays," Information Sciences, vol. 232, pp. 386-396, 2013.

[19] H. Huang, G. Feng, and J. Cao, "Robust state estimation for uncertain neural networks with time-varying delay," IEEE Transactions on Neural Networks, vol. 19, no. 8, pp. 1329-1339, 2008.

[20] T. Huang, "Robust stability of delayed fuzzy Cohen-Grossberg neural networks," Computers \&amp; Mathematics with Applications, vol. 61, no. 8, pp. 2247-2250, 2011.

[21] T. Huang, C. Li, S. Duan, and J. A. Starzyk, "Robust exponential stability of uncertain delayed neural networks with stochastic perturbation and impulse effects," IEEE Transactions on Neural Networks and Learning Systems, vol. 23, no. 6, pp. 866-875, 2012.

[22] G. C. Adam, B. D. Hoskins, M. Prezioso, F. Merrikh-Bayat, B. Chakrabarti, and D. B. Strukov, "3-D memristor crossbars for analog and neuromorphic computing applications," IEEE Transactions on Electron Devices, vol. 64, no. 1, pp. 312-318, 2017.

[23] F.-X. Wu, "Delay-independent stability of genetic regulatory networks," IEEE Transactions on Neural Networks, vol. 22, no. 11, pp. 1685-1693, 2011.

[24] Z. Yi, "Foundations of implementing the competitive layer model by Lotka-Volterra recurrent neural networks," IEEE Transactions on Neural Networks, vol. 21, no. 3, pp. 494-507, 2010.

[25] Z. Yi, L. Zhang, J. Yu, and K. K. Tan, "Permitted and forbidden sets in discrete-time linear threshold recurrent neural networks," IEEE Transactions on Neural Networks, vol. 20, no. 6, pp. 952-963, 2009.

[26] J. Yu, C. Hu, and H. Jiang, " $\alpha$-stability and $\alpha$-synchronization for fractional-order neural networks," Neural Networks, vol. 35, pp. 82-87, 2012.

[27] J. Yu, C. Hu, H. J. Jiang, and X. L. Fan, "Projective synchronization for fractional neural networks," Neural Networks, vol. 49, pp. 87-95, 2014.

[28] H. Zhang, J. Liu, D. Ma, and Z. Wang, "Data-core-based fuzzy min-max neural network for pattern classification," IEEE Transactions on Neural Networks, vol. 22, no. 12, pp. 2339-2352, 2011.
[29] H. Zhang, Y. Luo, and D. Liu, "Neural-network-based nearoptimal control for a class of discrete-time affine nonlinear systems with control constraints," IEEE Transactions on Neural Networks, vol. 20, no. 9, pp. 1490-1503, 2009.

[30] H. Zhang, T. Ma, G.-B. Huang, and Z. Wang, "Robust global exponential synchronization of uncertain chaotic delayed neural networks via dual-stage impulsive control," IEEE Transactions on Systems, Man, and Cybernetics, Part B: Cybernetics, vol. 40, no. 3, pp. 831-844, 2010.

[31] E. Devane and I. Lestas, "Delay-independent incremental stability in time-varying monotone systems satisfying a generalized condition of two-sided scalability," Automatica, vol. 76, pp. 1-9, 2017.

[32] Y. Wu, R. Lu, P. Shi, H. Su, and Z. Wu, "Adaptive output synchronization of heterogeneous network with an uncertain leader," Automatica, vol. 76, pp. 183-192, 2017.

[33] R. Naldi, M. Furci, R. G. Sanfelice, and L. Marconi, "Robust global trajectory tracking for underactuated VTOL aerial vehicles using inner-outer loop control paradigms," IEEE Transactions on Automatic Control, vol. 62, no. 1, pp. 97-112, 2017.

[34] A. Wu, L. Liu, T. Huang, and Z. Zeng, "Mittag-Leffler stability of fractional-order neural networks in the presence of generalized piecewise constant arguments," Neural Networks, vol. 85, pp. 118-127, 2017.

[35] C. P. Bechlioulis and G. A. Rovithakis, "Decentralized robust synchronization of unknown high order nonlinear multi-agent systems with prescribed transient and steady state performance," IEEE Transactions on Automatic Control, vol. 62, no. 1, pp. 123-134, 2017.

[36] I. E. Ebong and P. Mazumder, "CMOS and memristor-based neural network design for position detection," Proceedings of the IEEE, vol. 100, no. 6, pp. 2050-2060, 2012.

[37] K. C. Rahman, D. Hammerstrom, Y. Li, H. Castagnaro, and M. A. Perkowski, "Methodology and design of a massively parallel memristive stateful IMPLY logic-based reconfigurable architecture," IEEE Transactions on Nanotechnology, vol. 15, no. 4, pp. 675-686, 2016. 


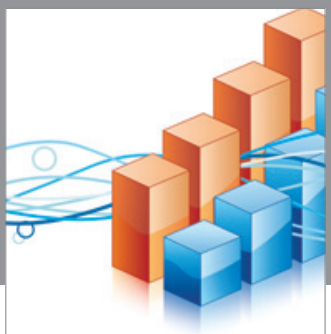

Advances in

Operations Research

vatem alat4

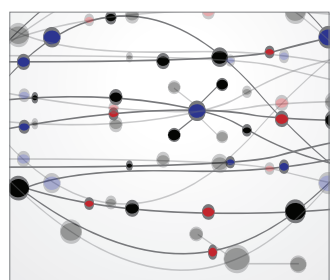

\section{The Scientific} World Journal
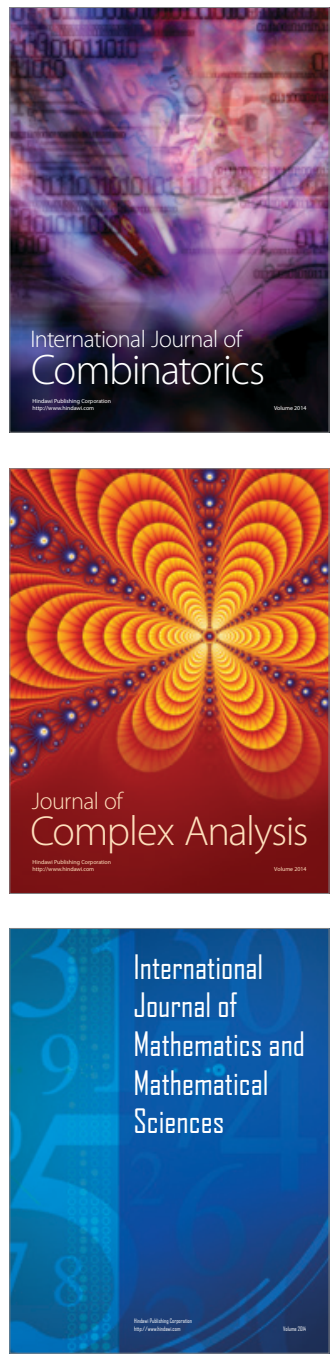
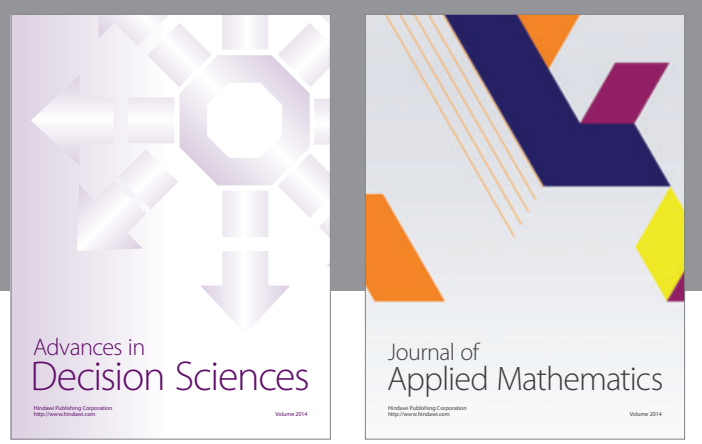

Algebra

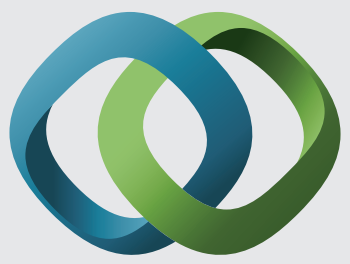

\section{Hindawi}

Submit your manuscripts at

https://www.hindawi.com
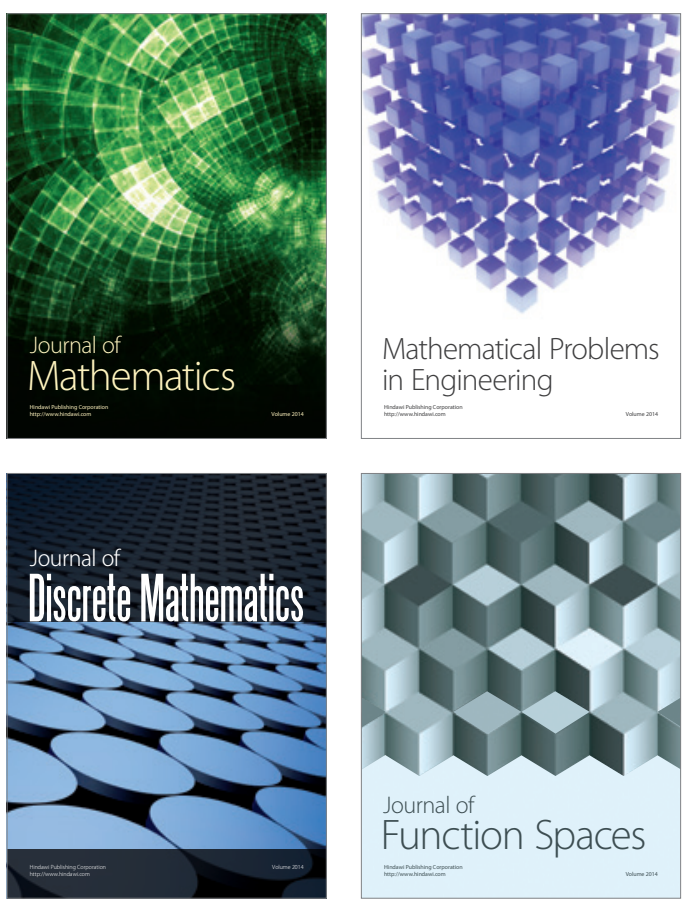

Mathematical Problems in Engineering
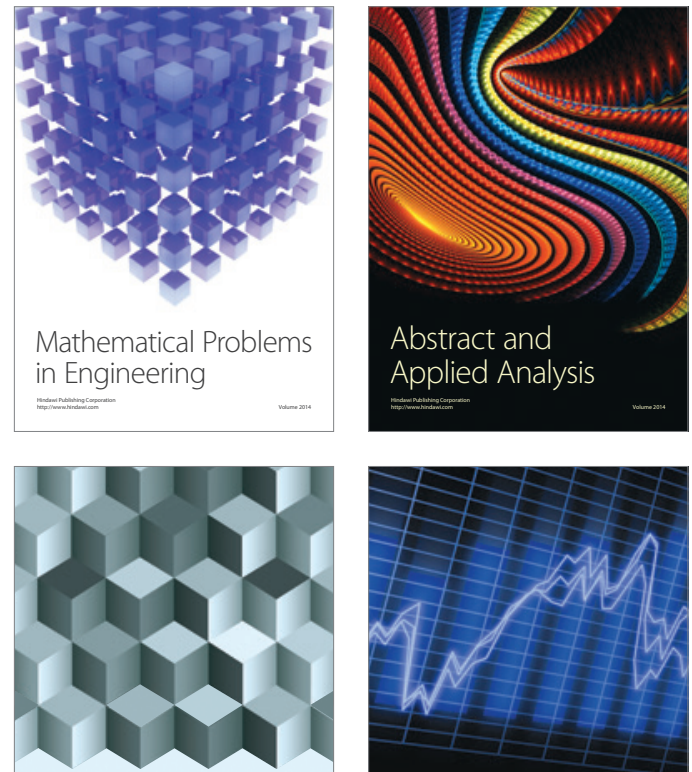

Journal of

Function Spaces

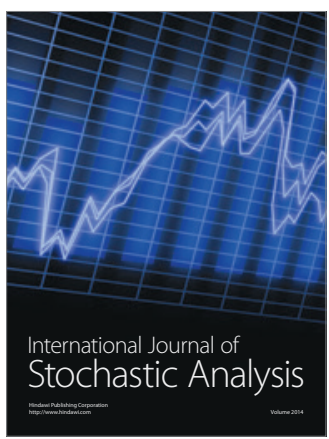

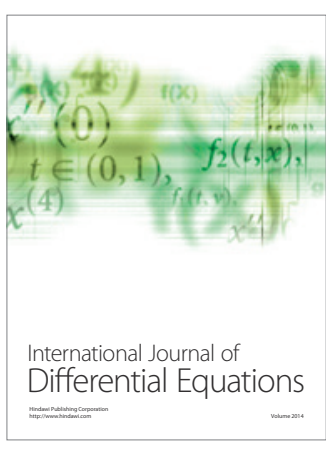
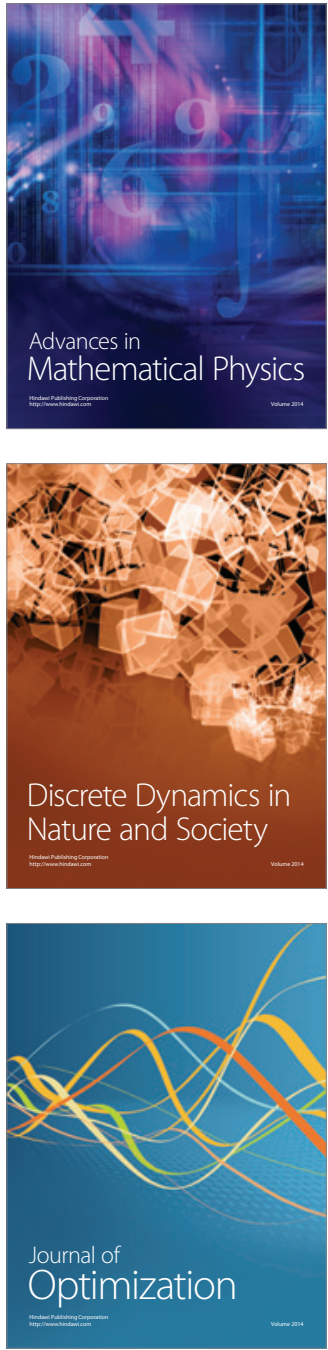